\title{
Serious infections during continuing treatment of acute lymphoblastic leukaemia
}

\author{
JACQUES NINANE AND JUDITH M CHESSELLS \\ Department of Haematology, The Hospital for Sick Children, London
}

SUMMARY Seventy-six of 168 children with acute lymphoblastic leukaemia, who had completed induction therapy and central nervous system prophylaxis, were each admitted to hospital at least once during subsequent treatment with an infectious illness. The time spent in hospital was calculated as a proportion of the total number of days at risk and had a mean value of one day in 75 . Sixty-three $(37 \%)$ children spent longer than this in hospital, particularly patients younger than 5 years and those on a more intensive protocol; a third year of treatment appeared to carry no significant additional risk of infection. Death due to infection occurred in $14(8.3 \%)$ children; these deaths were more common in younger patients and during the first 2 years of treatment and were mainly due to measles, septicaemia, or cytomegalovirus. These findings show that younger children and those receiving more intensive treatment are at greater risk of infection. The risk is not one of exotic infections, but of measles, a preventable disease.

Current treatment for acute lymphoblastic leukaemia (ALL) in childhood, although moderately aggressive, is given mainly on an outpatient basis in an attempt to allow the child and his family to enjoy as normal a life as possible.

However, infections requiring hospital treatment are a common complication of cytotoxic therapy, and may adversely affect the quality of life that is aimed for. Indeed the death rate from infection in first remission is still unacceptably high and in children presenting with ' standard risk' prognostic features it may even approach that from leukaemia. ${ }^{1}$

In an attempt to identify patients at risk of infections we have analysed the proportion of time spent in hospital by children undergoing continuing treatment for ALL.

\section{Patients and methods}

Between July 1973 and December 1977, 168 consecutive patients under age 15 years presenting with ALL and having completed remission induction and central nervous system prophylaxis (cranial irradiation and intrathecal methotrexate), were treated for 2 or 3 years with multiple-agent chemotherapy.

Any serious infective illness - defined as a febrile episode needing hospital treatment-was identified retrospectively and classified on the basis of review of clinical records. Days at risk were calculated from the end of cranial radiotherapy until either the end of chemotherapy or relapse or death in remission, and the proportion of this time spent in hospital because of infections was calculated for each patient. The study finished in July 1980 with a minimum follow-up period of $2 \frac{1}{2}$ years, at which time only one patient remaining in first complete remission was still on chemotherapy; the others had all stopped treatment.

\section{Results}

The patients comprised $75(45 \%)$ girls and $93(55 \%)$ boys of whom $93(55 \%)$ were younger than 5 years at the time of diagnosis. One hundred and sixteen patients were treated with a standard protocol, the Medical Research Council's UKALL II, III, IV, and $\mathrm{V},,^{1-3}$ and $52(31 \%)$ with adverse prognostic features were treated with an intensive protocol. ${ }^{4}$

Number of admissions and length of hospital stay. Each of $76(45 \%)$ children was admitted at least once during continuing treatment because of a febrile illness (Figure). These children spent a total of 1547 days in hospital during 139 separate admissions. The total number of patients-days at risk for the whole series of 168 patients was 116,156 , so that the mean proportion of this time spent in hospital with an infection was one day in $\mathbf{7 5}$ for the entire group. One hundred and six $(63 \%)$ patients spent a shorter time than this in hospital, and $62(37 \%)$ a longer time (maximum one day in 5-6). 


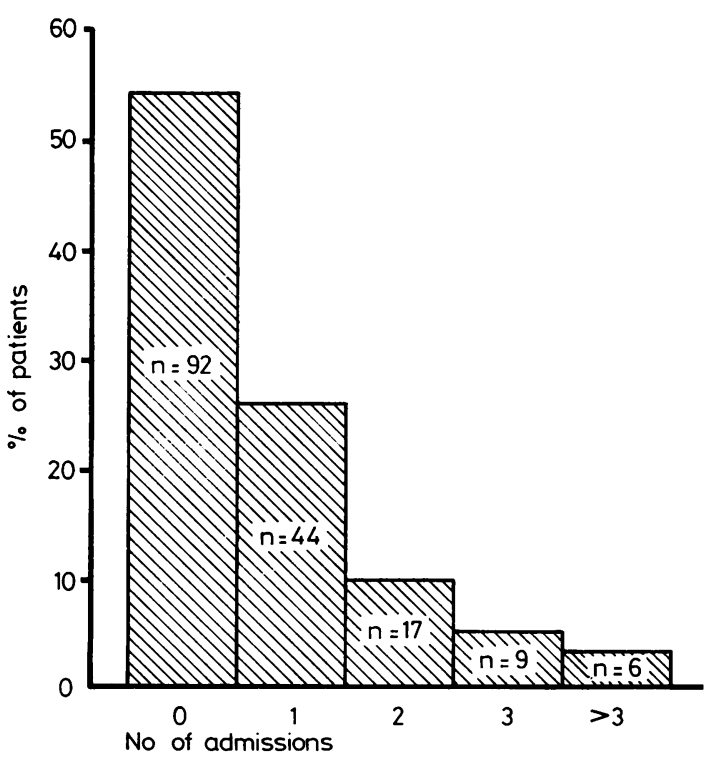

Figure Number of admissions

A higher proportion was found in children less than 5 years $(P<0.045)$ and in patients treated with an intensive protocol $(P<0.03)$ (Table 1). Although the number of admissions was higher in patients treated for more than 2 years, the proportion of time spent in hospital was not appreciably greater.

Reasons for admission. The diagnoses of the febrile illnesses which led to admission to hospital are shown in Table 2, together with the causes of death. Respiratory tract infections-lower and upper-were the most common, with 82 admissions $(59 \%$ of all admissions). Septicaemia was due to Gram-negative agents in 5 patients (Haemophilus influenzae non-B in 2, and one each of Escherichia coli, Klebsiella aeruginosa, and Pseudomonas aeruginosa) and to Gram-positive ones in 2 (Staphylococcus aureus and Clostridium sp.). One patient developed a retinitis

Table 1 Patients' characteristics

\begin{tabular}{|c|c|c|c|c|}
\hline & & No & Proportion & $P \dagger$ \\
\hline Age & $\begin{array}{l}<5 \text { years } \\
>5 \text { years }\end{array}$ & $\begin{array}{l}93 \\
75\end{array}$ & $\left.\begin{array}{l}0.021 \\
0.010\end{array}\right\}$ & $<0.045$ \\
\hline Sex & $\begin{array}{l}\text { Girls } \\
\text { Boys }\end{array}$ & $\begin{array}{l}75 \\
93\end{array}$ & $\left.\begin{array}{l}0.015 \\
0.015\end{array}\right\}$ & $>0.05$ \\
\hline Protocol & $\begin{array}{l}\text { Intensive } \\
\text { Ordinary }\end{array}$ & $\begin{array}{r}52 \\
116\end{array}$ & $\left.\begin{array}{l}0.026 \\
0.011\end{array}\right\}$ & $<0.03$ \\
\hline $\begin{array}{l}\text { Duration of } \\
\text { treatment }\end{array}$ & $\begin{array}{l}\leqslant 2 \text { years } \\
\$ 2 \text { years }\end{array}$ & $\begin{array}{l}88 \\
80\end{array}$ & $\left.\begin{array}{l}0.016 \\
0.012\end{array}\right\}$ & $>0.05$ \\
\hline
\end{tabular}

* Ratio of number of days in hospital to number of days at risk. $t \gamma^{2}$ test 1 degree of freedom.
Table 2 Reasons for admission-sites or organism

\begin{tabular}{llc}
\hline & $\begin{array}{c}\text { No of } \\
\text { admissions }\end{array}$ & Deaths \\
\hline LRTI* & 22 & 1 \\
Bacterial & 16 & 2 \\
Viral & 10 & - \\
$\begin{array}{l}\text { Pneumocystis carinii } \\
\text { Unknown cause }\end{array}$ & 22 & - \\
URTI $\dagger$ & 22 & 4 \\
Septicaemia & 7 & 1 \\
Varicella-zoster infection & 11 & 2 \\
Herpes simplex & 1 & - \\
Cytomegalovirus & 5 & - \\
Measles & 4 & - \\
Rubella & 1 & - \\
Meningitis & 3 & - \\
Osteomyelitis & 2 & - \\
Cellulitis & 7 & \\
Urinary tract infection & 2 & \\
Orchitis & 1 & \\
Appendicitis & 12 & \\
Pyrexia of unknown origin & 12 & \\
\hline
\end{tabular}

*Lower respiratory tract infection (bronchitis, pneumonia, bronchopneumonia, bronchiolitis, and lung abscess).

†Upper respiratory tract infection (otitis media, tonsillitis, rhinopharyngitis, laryngitis, oral candida).

and pneumonitis due to cytomegalovirus infection and is now blind. In $12(8.5 \%)$ patients there was no obvious clinical cause of infection and microbiological investigations were negative.

Deaths. The 14 deaths (Table 2) represent a mortality rate of $8 \cdot 3 \%$ for the entire group and a rate of $18 \cdot 4 \%$ for patients who were admitted. Death occurred more often in children under 5 years of age $(P<0.02)$ and during the first 2 years of chemotherapy: only 3 children died during the third year whereas 11 died during the first 2 years. The mortality for those treated in intensive protocols $(9.6 \%)$ was not significantly greater than for those on standard ones $(7 \cdot 8 \%)$.

Diseases with a high mortality rate were measles, viral pneumonias, septicaemia, and infections due to cytomegalovirus. The 2 patients who died from viral pneumonia may have had measles, since lung histology showed giant cells but immunofluorescence studies were not performed and the virus was thus not identified. If these were measles, the number of deaths due to this virus would be 5 ( $36 \%$ of children who died). The 4 patients who died from septicaemia ( $K$. aeruginosa, $H$. influenzae, Clostridium sp., $P$. aeruginosa) had had fever for longer than $\mathbf{4 8}$ hours before admission, were shocked when admitted, and were profoundly neutropenic $\left(<0.1 \times 10^{9} / 1\right.$ in 2 , and $<0.5 \times 10^{9} / 1$ in all). One of the patients with $H$. influenzae septicaemia had had peritonitis and a subphrenic abscess necessitating laparotomy and splenectomy during his remission induction. He was taking prophylactic penicillin. 


\section{Discussion}

Our results show that almost half the children who achieved a complete remission and completed a course of prophylaxis to the central nervous system were each admitted at least once because of an infectious illness during subsequent chemotherapy. Time spent in hospital had a mean proportion of one day in $\mathbf{7 5}$ days at risk for the group as a whole. However, most $(81 \%)$ children had no more than one hospital admission during continuing chemotherapy. Not surprisingly, patients receiving more intensive chemotherapy were more at risk of infection during continuing treatment as they were during remission induction. ${ }^{5}$

Children younger than 5 years had a higher risk of infection because chickenpox and measles-two potentially fatal diseases in patients receiving chemotherapy $-{ }^{67}$ are more common and upper respiratory tract infections more severe in young children.

Forty-three per cent of admissions were due to lower respiratory tract infections and in most of these an aetiological agent was not identified. Although routine bacteriological cultures were done in all cases on admission, a full screen for possible viral infections was not performed in all cases, and thus the incidence of viral infections in particular, might have been underestimated. In a recent multicentre analysis of incidence of pneumonia during treatment of ALL no agent was identified in $80 \%$ of cases. $^{8}$

In our series, death due to infection occurred in $8 \%$ of patients in remission. Simone et al. ${ }^{9}$ in 1972 reported remission fatalities of $16 \%$, and Craft et al. ${ }^{10}$ in 1977 found that infection caused the death in $18 \%$ of children in complete remission. The mortality rate in our series is unacceptably high in that 4 patients died from septicaemia, a complication which should be amenable to early recognition, treatment with antibiotics and, if indicated, granulocyte transfusions. These results stress the high mortality rate of measles.

No child who died from measles had been immunised. A history of measles vaccination was obtained in only $45 \%$ of the children older than 18 months at diagnosis who would have been eligible to receive measles vaccine, and a further $27 \%$ of children had a history and serological evidence of previous measles infection. Thus one-quarter of the patients were at high risk of this infection. Measles vaccination is contraindicated in the child receiving treatment for leukaemia ${ }^{11}$ but while some passive protection may be afforded by immune globulin, patients frequently have no history of contact and there remains no effective treatment for established infection. Sadly, with the continued poor uptake of vaccine in this country, it seems likely that such preventable deaths will continue. We are now attempting to reduce the risk of exposure to infection by encouraging vaccination of non-immune siblings and other close contacts.

By contrast the mortality rate from varicella was lower; treatment of the established disease has been available with adenine arabinoside and, more recently, with acyclovir ${ }^{12}$ and a varicella vaccine may ultimately be available. ${ }^{13}$

There were no more fatalities in children treated for 3 years with cytotoxic drugs than in those treated for 2 years. This is partly because pneumonia due to Pneumocystis carinii, which occurs late in the treatment, and which was the cause of death in 7 children in the Simone et al. series, ${ }^{9}$ is now easily recognised and responds either to co-trimoxazole or pentamidine. ${ }^{14}$

Quality of life is notoriously difficult to assess and measuring the proportion of time a child spends in hospital can provide only the crudest index of disability. A more sensitive and realistic index might be school absence which we have found in a recent survey to be very high in some children with ALL. ${ }^{15}$ The absence is dictated in part by the need to avoid contact with measles and varicella; the disastrous consequences of acquisition of these infections are illustrated in this paper.

Nevertheless, it is reassuring that, for the majority of children, particularly the older ones, treatment is completed with at most one hospital admission.

JN was supported by the Leukaemia Research Fund.

\section{References}

1 MacLennon I C M, Kay H E M, Festenstein M, Smith P G, et al. (Medical Research Council Working Party on Leukaemia in Childhood). Analysis of treatment in childhood leukaemia. II. Timing and the toxicity of combined 6-mercaptopurine and methotrexate maintenance therapy. $\mathrm{Br} J$ Haematol 1976; 33: 179-88.

2 MacLennon I C M, Peto J, Kay H E M (Medical Research Council Working Party on Leukaemia in Childhood). Analysis of treatment in childhood leukaemia. V. Advantage of reduced chemotherapy during and immediately after cranial irradiation. $B r J$ Cancer 1977 ; 36: 625-33.

3 Rapson N T, Cornbleet M A, Chessells J M, Bennett A J, Hardisty R M. Immunosuppression and serious infections in children with acute lymphoblastic leukaemia: a comparison of three chemotherapy regimes. $\mathrm{Br} \mathrm{J} \mathrm{Haematol}$ $1980 ; 45$ : 41-52.

4 Chessells J, Hardisty R M, Rapson N T, Greaves M F. Acute lymphoblastic leukaemia in children: classification and prognosis. Lancet 1977; ii: 1307-9.

5 Chessells J M, Leiper A D. Infection during remission induction in childhood leukaemia. Arch Dis Child 1980; 55: 118-23. 


\section{Ninane and Chessells}

${ }^{6}$ Pullan C R, Noble T C, Scott D, Wisniewski K, Gardner P S. Atypical measles infections in leukaemic children on immunosuppressive treatment. $\mathrm{Br} \mathrm{Med} J$ 1976; i: 1562-5.

- Feldman S, Hughes W T, Daniel C B. Varicella in children with cancer: seventy seven cases. Pediatrics 1975; 56: 388-92.

8 Siegel S, Nesbit M E, Baehner R, Sather H, Hammond D. Pneumonia during therapy for childhood acute lymphoblastic leukaemia. Am J Dis Child 1980; 134: 28-34.

9 Simone J V, Holland E, Johnson W. Fatalities during remission of childhood leukemia. Blood 1972; 39: 759-70.

10 Craft A W, Reid M M, Bruce E, Kernahan J, Gardner P S. Role of infection in the death of children with acute lymphoblastic leukaemia. Arch Dis Child 1977; 52: 752-7.

11 Mitus A, Holloway A, Evans A E, Enders J F. Attenuated measles vaccine in children with acute leukaemia. $A m \mathrm{~J}$ Dis Child 1962; 103: 413-8.
12 Schaeffer H J, Beauchamp L, de Miranda P, Elion G B. 9-(2-Hydroxyethoxymethyl) guanine activity against viruses of the herpes group. Nature $1978 ; 272$ : 583-5.

13 Asano $Y$, Nakayama $H$, Yazaki $T$, et al. Protection against varicella in family contacts by immediate inoculation with live varicella vaccine. Pediatrics 1977 ; 59: 3-7.

14 Hughes W T. Treatment of pneumocystis carinii pneumonitis. $N$ Engl J Med 1976; 295 : 726-7.

15 Eiser C. How leukaemia affects a child's schooling. $\mathrm{Br} J$ Soc Clin Psychol 1980; 19: 365-8.

Correspondence to Dr J M Chessells, Department of Haematology, The Hospital for Sick Children, Gt Ormond Street, London WC1N 3JH.

Received 24 November 1980

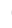

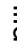

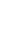
(a) 的 จิจ œ 